\title{
Intercomparación en Mediciones Superficiales
}

\section{Intercomparison in Surface Measurements}

Presentación: 31/10/2018

Aprobación: 05/02/2019

\section{Nancy Brambilla}

Centro de Investigación y Transferencia en Metrología CEMETRO -Universidad Tecnológica Nacional, Facultad Regional Córdoba.

nbrambilla@frc.utn.edu.ar

\section{Daniel Brusa}

Centro de Investigación y Transferencia en Metrología CEMETRO -Universidad Tecnológica Nacional, Facultad Regional Córdoba.

dhbrusa@gmail.com

\section{Juan Caselles}

Centro de Investigación y Transferencia en Metrología CEMETRO -Universidad Tecnológica Nacional, Facultad Regional Córdoba.

caselles_juan@hotmail.com

\section{Carlos Mas}

Centro de Micro y Nanoscopía de Córdoba - CEMINCO (CIQUIBIC-CIBICI-INIMEC-CONICET- UNC). carlos@fcq.unc.edu.ar

\section{Clemar Schurrer}

Centro de Investigación y Transferencia en Metrología CEMETRO -Universidad Tecnológica Nacional, Facultad Regional Córdoba.

cschurrer@gmail.com

\section{Resumen}

La trazabilidad en mediciones superficiales de micro y nanoestructuras es condición ineludible para garantizar la calidad en proyectos de innovación, investigación y desarrollo en el espectro disciplinar que abarca las ciencias químicas, biológicas, de materiales, siendo además un requisito para mediciones de rugosidad en la industria. La confiabilidad metrológica (ISO 10012) presupone el uso de instrumentos calibrados, patrones trazables, procedimientos de medición validados y balances de incertidumbre. Estos requerimientos no son 
habituales en microscopía y representa una debilidad a subsanar. En este trabajo se presentan los resultados de la intercomparación de mediciones de textura superficial en patrones 2D y 3D con cuatro tipos de instrumentos: Rugosímetro, Microscopio Confocal, Microscopio de Fuerza Atómica y Microscopio Electrónico de Barrido. Las evaluaciones de conformidad (ISO 17043) mediante la comparación de los errores normalizados (En), que ponderan los efectos sistemáticos y sus incertidumbres, convalidan el uso de los microscopios como instrumentos metrológicos.

Palabras claves: Microgeometría, Nanogeometría, Rugosidad, Microscopía, Metrología Superficial, Conformidad Metrológica, Intercomparación

\begin{abstract}
Innovation, research and development projects that include surface measurements of micro and nanostructures must guarantee the traceability of them. The disciplinary spectrum that includes this type of measurements goes from chemical and biological sciences to the engineering of materials. The traceability is also a requirement for surface roughness in industry. Metrological reliability (ISO10012) presupposes the use of calibrated instruments, traceable patterns, validated measurement procedures and uncertainty balance. These requirements do not apply in microscopy, being a weakness to correct. In this paper we present the results of the intercomparison of surface texture measurements in 2D and 3D patterns with four types of instruments: Contact Roughness, Confocal Microscope, Atomic Force Microscope and Scanning Electron Microscope. The conformity assessments (ISO 17043), through the standardized errors (En) that weigh the systematic effects and their uncertainties, validate the use of microscopes as metrological instruments.
\end{abstract}

Keywords: Microgeometry, Nanogeometry, Roughness, Microscopy, Surface Metrology, Metrological Compliance, Intercomparison.

\title{
Introducción
}

La exactitud de las mediciones de microgeometría y nanogeometría superficial tienen una relevancia creciente por su impacto en un amplio espectro de actividades y desarrollos innovadores. Desde los ensayos y mediciones normalizadas de rugosidad superficial requeridas en la industria metalmecánica hasta la caracterización cristalográfica de los nuevos materiales nanoestructurados para funciones específicas, la calidad metrológica de la textura superficial es un requerimiento primordial.

Las irregularidades de una superficie se clasifican según la frecuencia espacial. La totalidad del espectro de irregularidades se divide en tres regiones: alta, media y baja frecuencia, que se denominan en los respectivos rangos como: rugosidad, ondulación y error de forma [ISO 4287-1997, D. Whitehouse 2011]. Los errores de forma, asociados con la variación en tamaño de una pieza, paralelismo, planitud, conicidad, redondez y cilindricidad, pueden 
medirse con instrumentos convencionales de mano o con equipos especiales como máquina de medir en coordenadas, con exactitudes acordes a las tolerancias estipuladas. La ondulación y la rugosidad propiamente dicha están en general asociadas a los procesos de fabricación y acabado de superficies y se relevan con rugosímetros.

Las mediciones de rugosidad superficial en piezas de ingeniería en la industria aeronáutica y metalmecánica, para aplicaciones en superficies de levas, vástagos y asientos de válvulas, caras de pistón, bielas, cigüeñal; así como la calibración de patrones de rugosidad a nivel industrial, son prácticas normalizadas usando rugosímetros y/o perfilómetros por contacto, en base a la norma ISO 4288:98 y otras [Vorburger, 1990]. Se informan los resultados a través de los parámetros característicos de rugosidad según la escala Ra, Rz y Rt [Leach 2014]. Análisis más detallados como los que usan en la medición de proyectiles requieren de otros parámetros [J. Song 2005].

Dependiendo de la funcionalidad de las superficies, los controles de rugosidad requieren distintos métodos de medición [Whitehouse, 1997]. Esta particularidad ha hecho que se deban intercomparar y validar diversas técnicas aplicadas sobre una misma superficie sobre todo cuando existen rangos extensos de escalas (ie. nano a micro) involucradas [N. Jouini 2009; M. Chand et al. 2011]. Los avances en las diversas áreas de aplicación de la micro y de la nanotecnología, requiere mediciones que deben realizarse en muchos casos sin contacto y con instrumental óptico, como microscopios de distinto tipo y escáneres; son ejemplo la rugosidad representada como un factor biológico a escala molecular que afecta el modo en que las bacterias se adhieren en la superficie. Las aplicaciones en el área de la medicina abarcan también la caracterización de la rugosidad de los implantes osteointegrados [Rama Krishna Alla et al. 2011; F. Ruppa et al. 2018] y en córneas [C. Jumelle et al. 2017].

Para asegurar la calidad de los resultados, las mediciones deben ser metrológicamente confiables, es decir deben sustentarse sobre una cadena de trazabilidad a patrones de referencia internacionales (J. Petzing 2010). En el área de la microscopía, es ésta una carencia detectada en nuestro medio ya que los microscopios tanto Confocales, como AFM y SEM en uso, no están calibrados, no tienen patrones de referencia o los tienen pero fuera de vigencia. En este sentido para el caso del AFM una alternativa es referir las mediciones longitudinales al patrón de longitud interferométrico (Dirscherl 2005) mientras que para el caso del microscopio Confocal existen algunas técnicas descriptas en Giusca et al. (2012).

El objetivo principal del presente trabajo es validar el uso de microscopios como instrumentos de medición de rugosidad superficial, para lo cual se propone realizar una intercomparación de los resultados obtenidos con microscopio Confocal, AFM, SEM y con rugosímetro de laboratorio sobre diversos patrones de forma tridimensional. En la sección 2 se indica la metodología normalizada para la evaluación de conformidad (ISO 17043) y el detalle de los instrumentos y patrones utilizados. En la sección 3 se presentan los resultados de las mediciones y la intercomparación de los parámetros superficiales ensayados y se interpretan y analizan los indicadores propuestos. Por último en la sección 4 se presentan las conclusiones y las líneas de acción futuras.

\section{Materiales y Métodos}

\subsection{Equipos}

Los equipos utilizados se presentan a continuación detallando sus principales características y especificaciones técnicas. En cada uno de ellos se realizaron las mediciones de los diferentes patrones, respetando los rangos y las posibilidades físicas de cada equipo (ver 
Figura 1)

Rugosímetro por Contacto Taylor Hobson: modelo TalySurf S2, radio palpador $2 \mu \mathrm{m}$, transductor inductivo, excursión $130 \mathrm{~mm}$, rango en altura $1 \mathrm{~mm}$, Resolución $16 \mathrm{~nm}$. Centro de Metrología (CEMETRO) UTN-FRC.

- Microscopio Confocal LEXT OLS4000: Área de escaneo 10x10mm Rango en altura $1 \mathrm{~mm}$ Resolución $1 \mathrm{~nm}$. Laboratorio de Microscopía Electrónica y Análisis por Rayos X (LAMARX) FaMAF-UNC.

- $\quad$ Microscopio de Fuerza Atómica Bruker Innova (AFM) Área de escaneo 100x100 $\mu \mathrm{m}$. Rango en altura $7 \mu \mathrm{m}$ Resolución 0,2nm. Centro de Metrología (CEMETRO) UTN-FRC.

Microscopio Electrónico de Barrido Zeiss Sigma 300 (SEM) Resolución 1nm. Laboratorio de Microscopía Electrónica y Análisis por Rayos X (LAMARX) FaMAF-UNC.
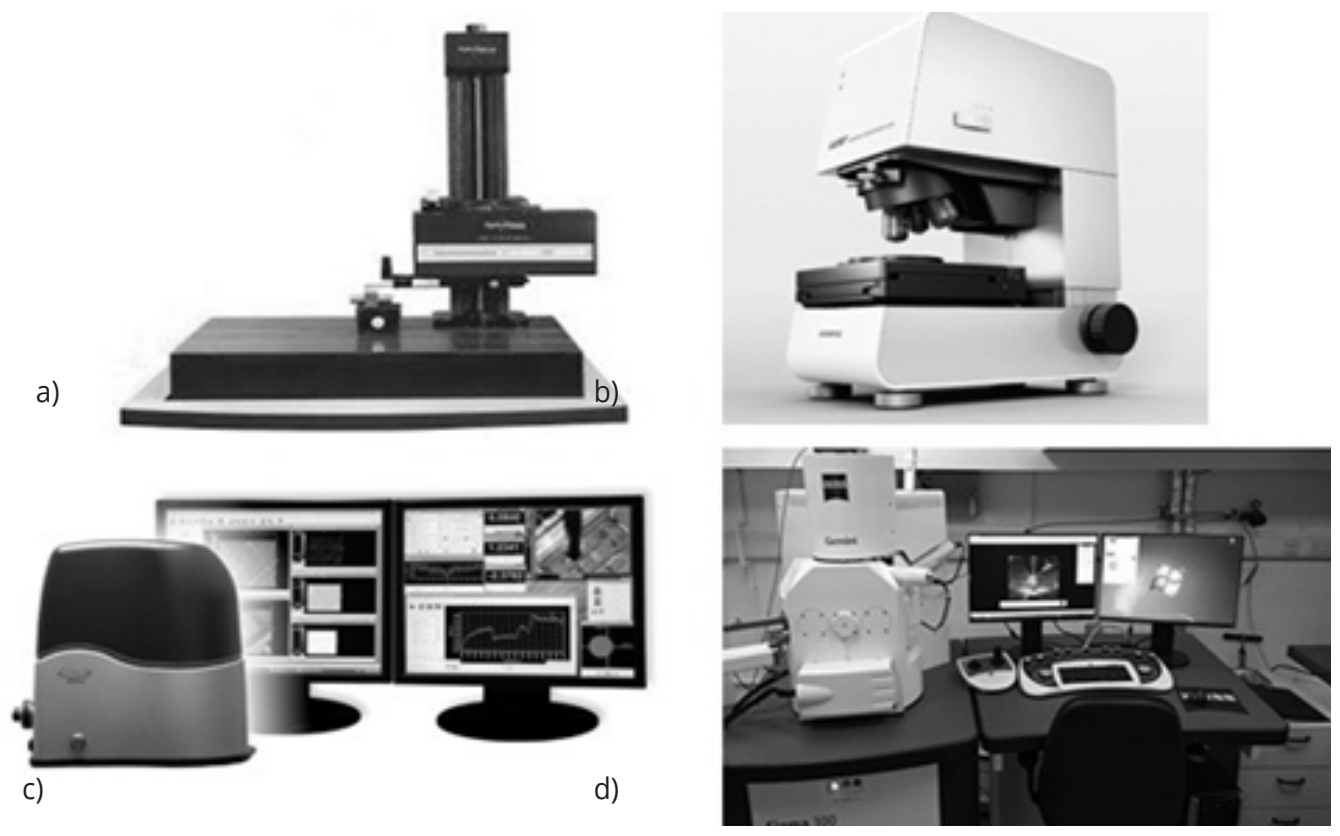

Figura 1: a) Rugosímetro, b) Confocal, c) AFM, d) SEM.

\subsection{Patrones}

Se usaron patrones del tipo grillas, escala de vidrio, patrón de ranuras y patrones de rugosidad de diferentes características, que se listan a continuación:

- $\quad$ Escala de vidrio lineal Mitutoyo. Longitud 50mm Resolución 0,1 mm

- $\quad$ Patrón de ranuras KMT 2060 Halle. Valores Nominales: $1 \mu \mathrm{m}, 4 \mu \mathrm{m}$ y $9 \mu \mathrm{m}$

- $\quad$ Patrón de rugosidad de vidrio perfil regular PGN3 Mahr-Perthen

- $\quad$ Patrón de rugosidad de acero perfil irregular RNDHZ Hommel-Etamic

- $\quad$ Patrón de grilla VGRP Bruker Surface Topography.

\subsection{Metodología}

La metodología empleada para el relevamiento de los patrones es similar con todos los equipos y consiste en realizar un escaneado del plano XY y las variaciones en altura y 
profundidad son medidas en el eje Z. Si bien los equipos utilizados presentan diferentes características y especificaciones técnicas, es posible utilizarlos para la medición de los respectivos patrones siempre que se respeten los rangos y las posibilidades físicas de cada uno. Por ejemplo, el tamaño de la muestra es un condicionante en el AFM por su reducido rango $(0,1 \mathrm{~mm} \times 0,1 \mathrm{~mm})$.

El procesamiento de los datos o resultados se realiza tomando como base la norma ISO/ IEC 17043: 2010: Evaluación de la Conformidad -Requisitos generales para los ensayos de aptitud, Anexo B - Métodos Estadísticos para Ensayos de Aptitud. En la misma se detallan los pasos fundamentales para los ensayos de aptitud, según:

a) Se determinan los valores asignados y su incertidumbre por medición para cada patrón en varios equipos. Se toma como medida de referencia los resultados de medición del rugosímetro, dado que el equipo tiene trazabilidad a patrones nacionales.

b) se realizan los cálculos estadísticos de desempeño para cada uno, en base a los resultados cuantitativos de cada equipo, obteniendo el índice "En" para cada comparación.

c) se realiza la evaluación del desempeño según el índice "En" para cada comparación, con el criterio establecido en la norma, esto es valor de En $\leq 1$ indica desempeño "satisfactorio".

Cálculo de En:

Este indicador estadístico se calcula como:

$$
E_{n}=\frac{\left|x-x_{R}\right|}{\sqrt{U_{x}+U_{R}}}
$$

Donde $\mathrm{x}$ y xR son el valor medido mediante una de las técnicas y el valor de referencia, y Ux y UR representan las incertidumbres respectivas.

\section{Resultados y Discusión}

Se procede con las mediciones relevando distintas zonas sobre las muestras y patrones previamente acondicionados. Se informan los valores promedios y sus incertidumbres, que se estima con mayor o menor detalle según el instrumental utilizado, incluyendo en todos los casos la variabilidad del mensurando, ponderada por la desviación estándar experimental de la media (tipo A) y las fuentes asociadas al instrumental y/o técnica de medición (tipo B). Las mediciones con rugosímetro por contacto tienen una evaluación de incertidumbre más rigurosa dado que la técnica está acreditada y respaldada por ensayos de aptitud realizados en el marco del servicio de calibración de patrones de rugosidad.

Las Capacidades de Medición y Calibración (CMC) o Mejores Capacidades de Medición (MCM) de los servicios acreditados del CEMETRO por el Organismo Argentino de Acreditación (OAA) y supervisado por el Servicio Argentino de Calibración del INTI (INTI$\mathrm{SAC}$ ) están publicadas en las páginas Webs de los Organismos.

Sobre cada patrón medido se hacen los correspondientes análisis de resultados ponderando las limitaciones y los hallazgos.

En el apartado 3.6 se discuten los resultados del ensayo de intercomparación en base a los 
criterios, términos y definiciones de la Norma ISO/IEC 17043 -Evaluación de Conformidad. Requisitos Generales para los Ensayos de Aptitud- para concluir sobre la fiabilidad de las mediciones obtenidas con los diferentes instrumentos sobre los patrones.

\subsection{Patrón de Ranuras KNT2060}

Este patrón específico de alta calidad metrológica se utiliza habitualmente para controlar variaciones en altura y profundidad calibrando principalmente el eje $\mathrm{Z}$ de rugosímetros.

\begin{tabular}{|l|l|l|l|}
\hline \multicolumn{4}{|c|}{ Valores de $\mathbf{R}_{\mathrm{t}} \pm \mathbf{U}(\boldsymbol{\mu m})$} \\
\hline KNT 2060 & Ranura $\mathbf{1} \boldsymbol{\mu m}$ & Ranura $\mathbf{4} \boldsymbol{\mu m}$ & Ranura $\mathbf{9} \boldsymbol{\mu m}$ \\
\hline Rugosímetro & $1,05 \pm 0,07$ & $3,9 \pm 0,3$ & $8,9 \pm 0,6$ \\
\hline Confocal & $1,02 \pm 0,20$ & $3,9 \pm 0,2$ & $9,2 \pm 0,3$ \\
\hline AFM & $1,02 \pm 0,05$ & $3,70 \pm 0,05$ & - \\
\hline
\end{tabular}

Tabla 1: Valores de Rt del KNT2060 medido con Rugosímetro, Microscopio Confocal y AFM
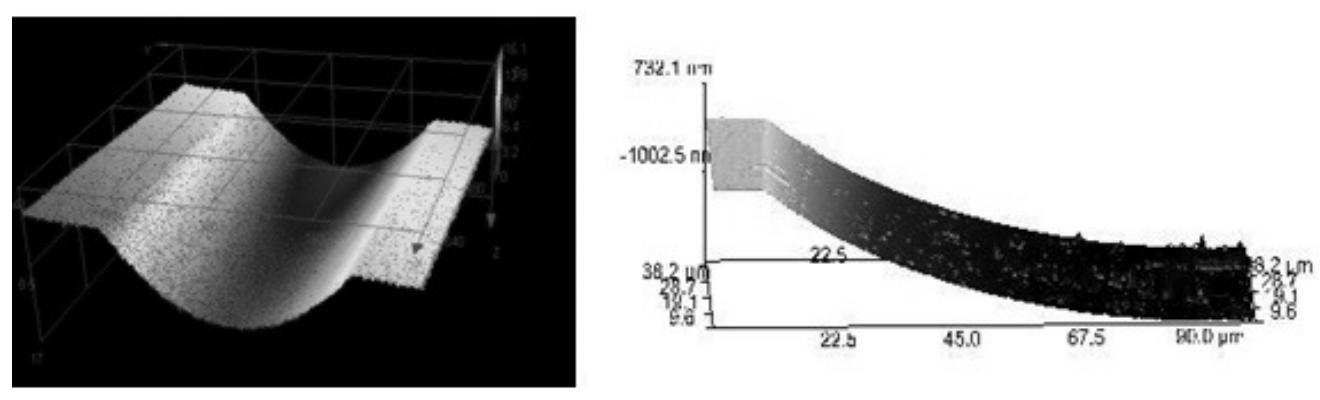

Figura 2: Imagen del Patrón KNT2060 Ranura de 9 um obtenida con el Confocal (a) y AFM (b) 


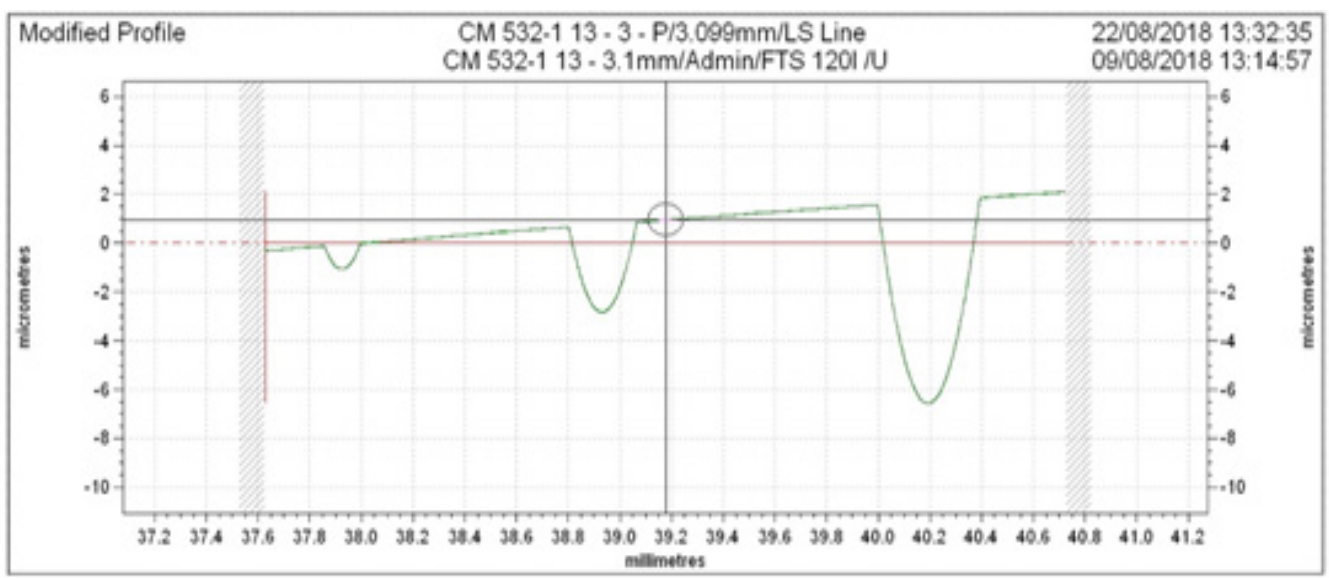

Figura 3: Perfil del Patrón KNT 2060 con las 3 ranuras obtenido con el Rugosímetro

Los valores expresados en la Tabla 1, que muestran el parámetro de rugosidad definido como Rt, corresponden al valor promedio de 10 mediciones distribuidas en diferentes áreas de la muestra. Se observa un muy buen acuerdo entre las mediciones del Rugosímetro y el Confocal. El AFM coincide en la ranura de $1 \mu \mathrm{m}$. En las figuras 2 y 3 se presenta los datos primarios con algunas de las imágenes y perfiles obtenidos.

La limitación del AFM, en cuanto al tamaño de la muestra por el rango XY, se pone en evidencia en las otras dos ranuras (4 y $9 \mu \mathrm{m}$ ). La ranura de $4 \mu \mathrm{m}$ se encuentra en el límite del rango de funcionamiento del instrumento e impide obtener una imagen completa del perfil. Si se utilizan dos imágenes superpuestas, es posible determinar aunque con dificultades el vértice del valle y con ello determinar la profundidad de la ranura de $4 \mu \mathrm{m}$. No es posible medir la ranura de $9 \mu \mathrm{m}$ con el AFM.

La información suministrada por cada equipo tiene sus particularidades; la que ofrece el AFM permite observar detalles locales del patrón, mientras el rugosímetro integra los valores primarios dando una información global. La Figura 4 muestra algunos de los perfiles obtenidos mediante AFM donde se pueden ver las variaciones que existen entre los diferentes perfiles dando cuenta de lo expresado previamente. 


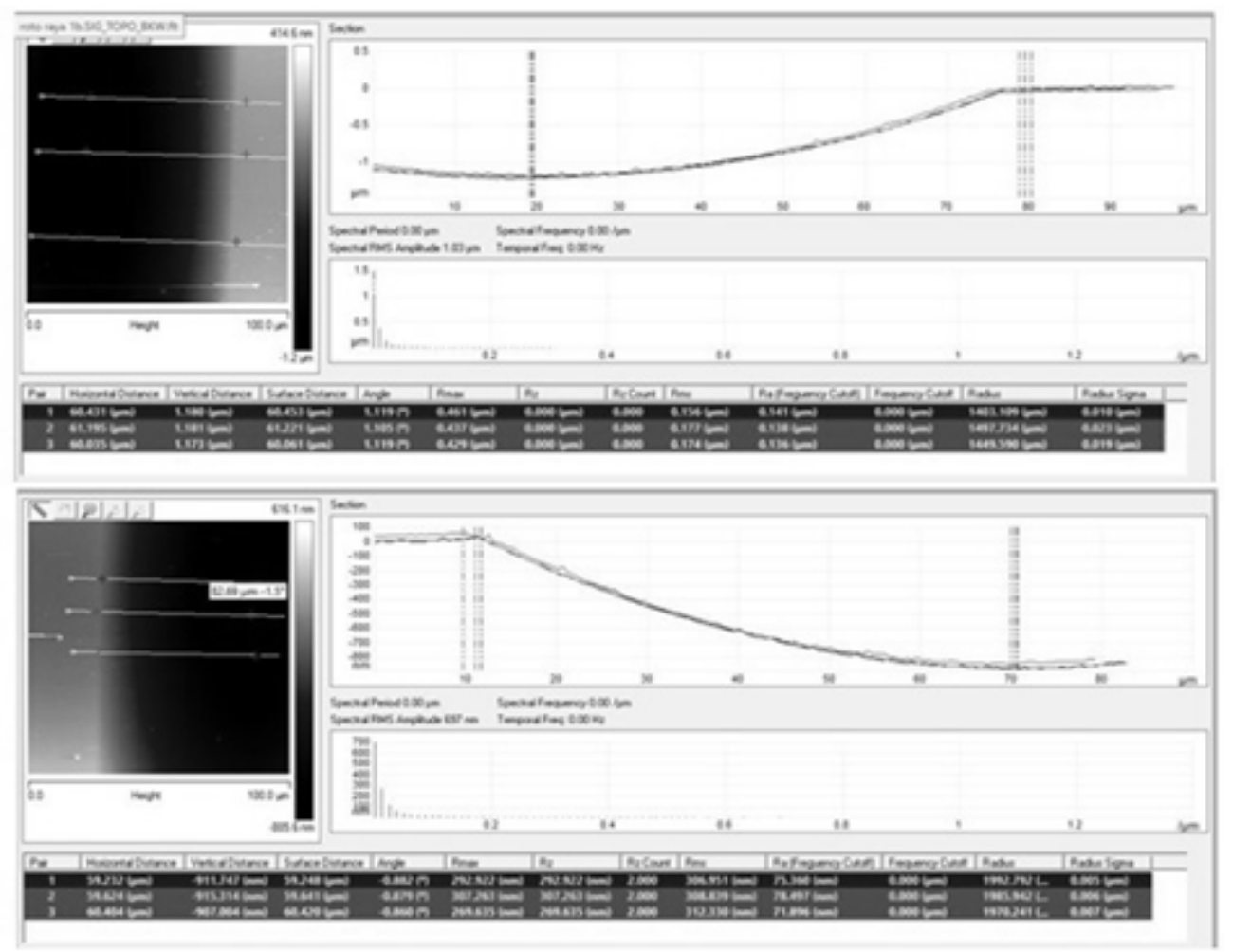

Figura 4: Imagen obtenida con el AFM del Patrón KNT2060

La ranura de $4 \mu \mathrm{m}$ tiene un ancho de $0,3 \mathrm{~mm}$ por lo que supera el límite del rango XY del AFM, este es el principal motivo de la diferencia observada en los resultados con respecto a los otros dos métodos (ver Figura 5).

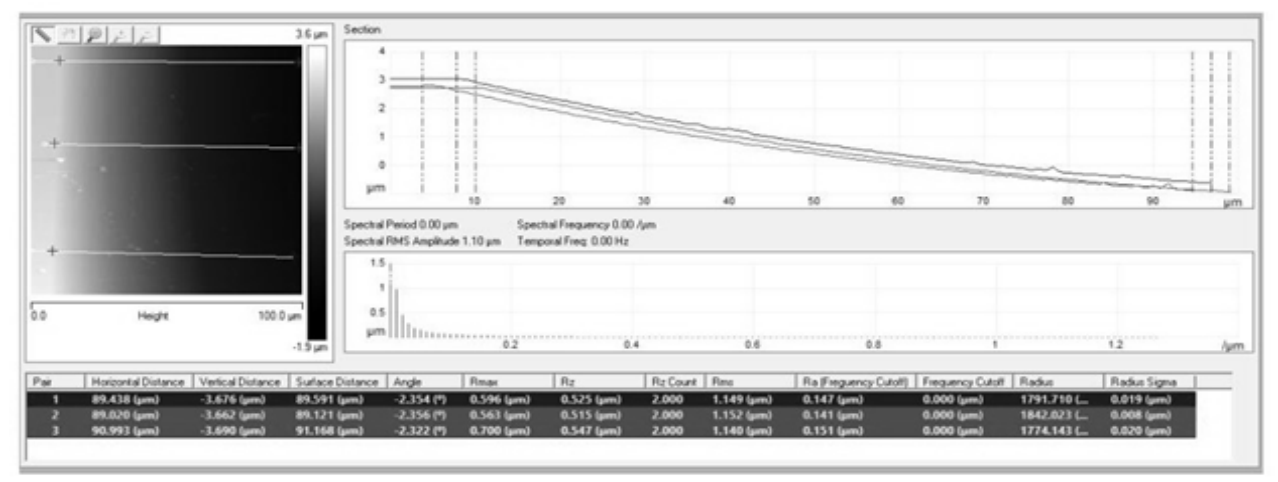

Figura 5: Imagen obtenida del Patrón KNT2060 Ranura 0,4 um con el AFM 
Observando los perfiles de las ranuras se determina que tienen una forma funcional característica de una parábola, que al ser analizada de manera más detallada y realizando un ajuste de función cuadrática se obtiene un muy buen acuerdo, con una correspondencia dentro de los 10 nm, según se aprecia en la Figura 6.
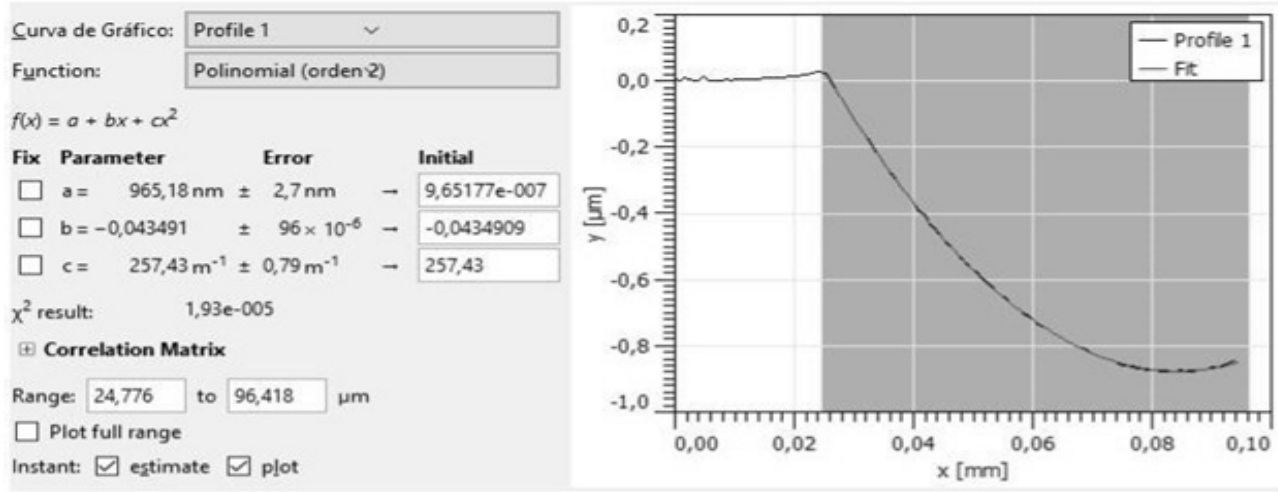

Figura 6: Perfil de una ranura del Patrón KNT2060 y ajuste de una función cuadrática

\section{Patrón de Rugosidad PGN3}

\subsection{Patrón de rugosidad PGN3}

Las características propias del patrón PGN3 permiten realizar mediciones de parámetros de rugosidad y de ondulación. La Tabla 2 presenta los resultados obtenidos y su consistencia. Los órdenes dispares de las incertidumbres dan cuenta de las capacidades de medición de cada equipo.

\begin{tabular}{|c|c|}
\hline \multicolumn{2}{|r|}{ Valores de $\mathbf{R}_{\mathrm{t}} \pm \mathbf{U} \mu \mathrm{m}$} \\
\hline \multicolumn{2}{|r|}{ PGN3 } \\
\hline Rugosímetro & $3,4 \pm 0,2$ \\
\hline AFM & $3,52 \pm 0,05$ \\
\hline
\end{tabular}

Tabla 2: Valores de Rt del PGN3 medido con Rugosímetro y Microscopio AFM

Los perfiles obtenidos en diferentes zonas del patrón se promedian y los resultados sepresentan en la Tabla 2. Un ejemplo del tipo de perfil obtenido se puede apreciar en la Figura 7; la gráfica superior es un perfil obtenido con el Rugosímetro con una sucesión de picos y valles regulares, en la parte inferior una imagen del AFM en la cual sólo se puede obtener 
un pico y un valle por cada imagen, y en esa imagen, el trazado de tres perfiles y la medición de las alturas.

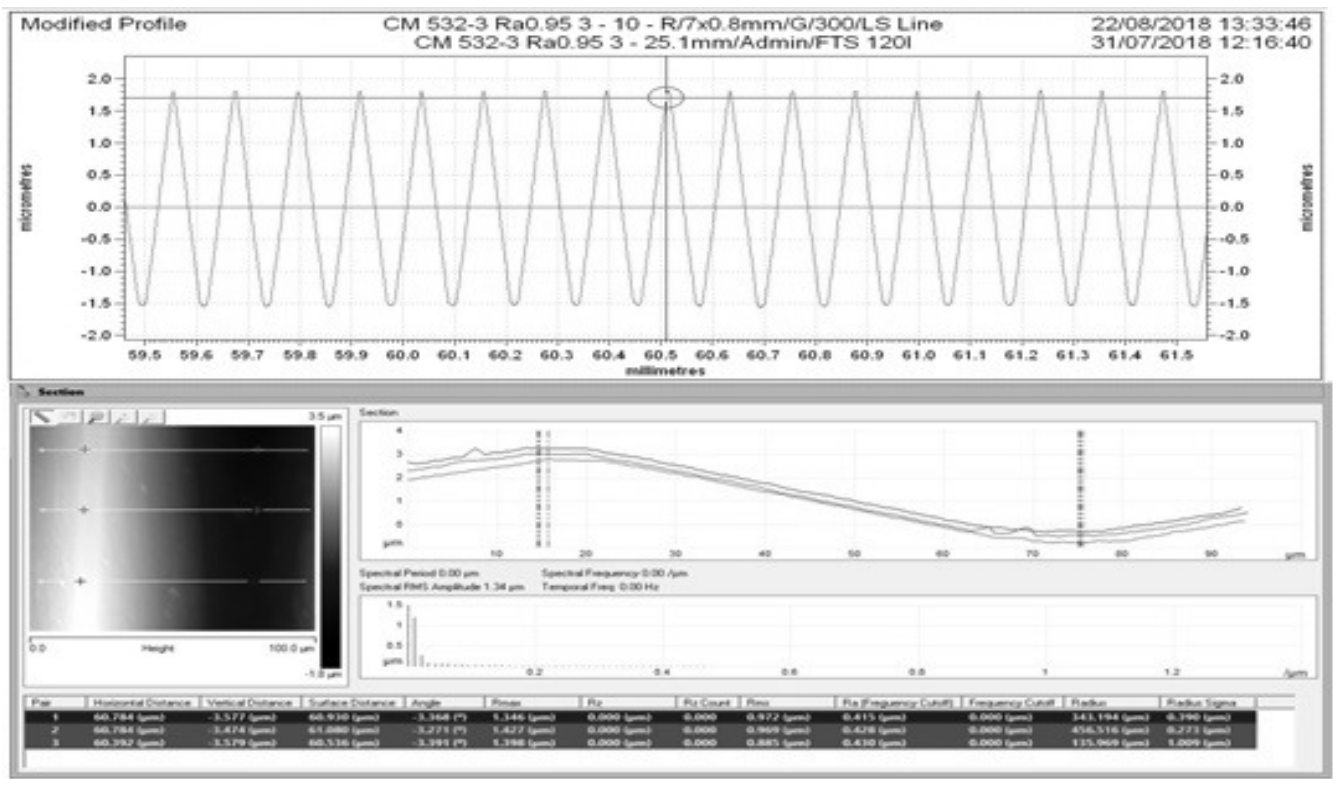

Figura 7: Imagen y Perfil del Patrón PGN3 obtenida por Rugosímetro y AFM

\subsection{Patrón de Rugosidad RNDH2}

Este patrón es fabricado en acero y muestra una estructura irregular muy utilizada para la determinación de parámetros de rugosidad. La Tabla 3 muestra el solapamiento de los resultados obtenidos sobre el patrón RNDH2.

\begin{tabular}{|l|l|}
\hline \multicolumn{2}{|c|}{ Valores de $\mathbf{R}_{\mathrm{t}} \pm \mathbf{U} \mu \mathrm{m}$} \\
\hline \multicolumn{2}{|c|}{ RNDH2 $^{2}$} \\
\hline Rugosímetro & $3,4 \pm 0,2$ \\
\hline AFM & $3,35 \pm 0,05$ \\
\hline
\end{tabular}

Tabla 3: Valores de Rt del RNDH2 medido con Rugosímetro y Microscopio AFM

La Figura 8 muestra la característica ondulación del patrón RNDH2 obtenida en el microscopio AFM y el rugosímetro, observándose que sobre las ondulaciones principales tiene montado una ondulación de mayor frecuencia y una amplitud del orden de $300 \mathrm{~nm}$. 


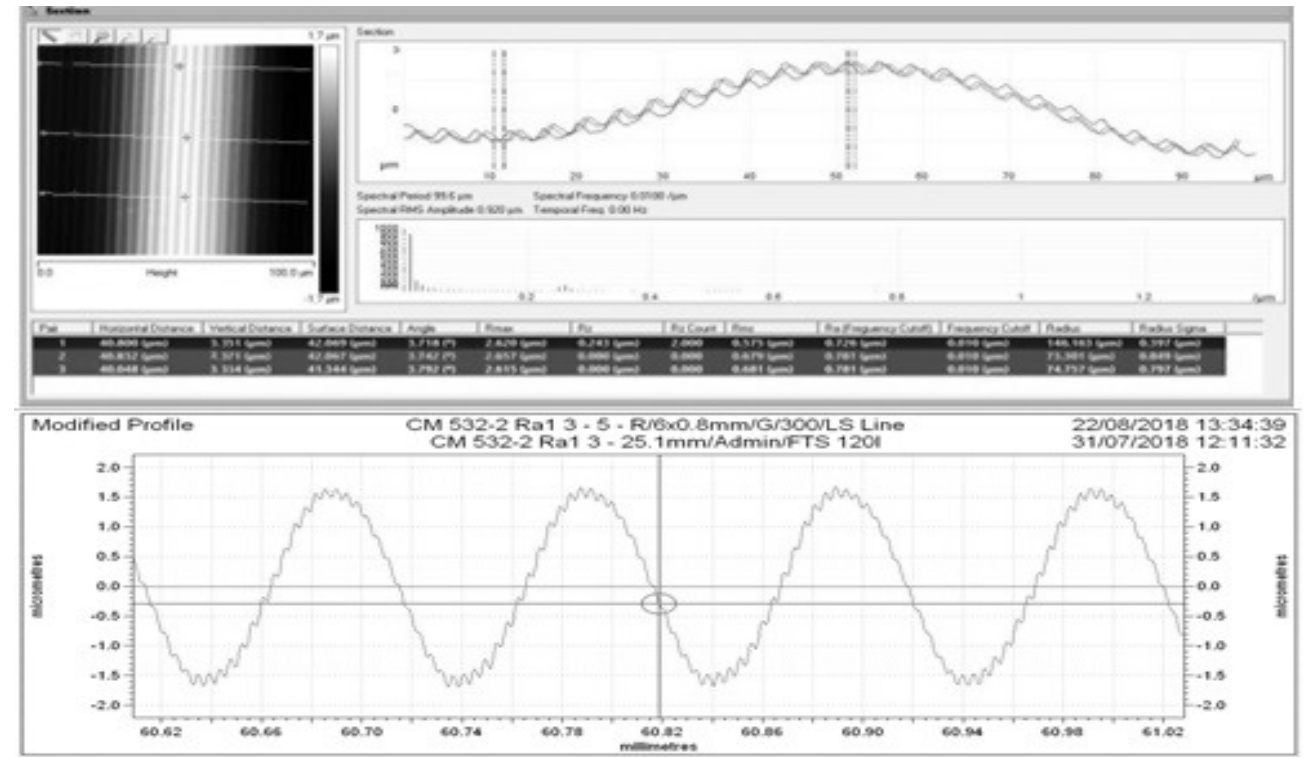

Figura 8: Imagen y Perfil del Patrón RNDH2 obtenida por AFM (superior) y Rugosímetro (inferior)

\subsection{Escala de vidrio lineal}

La escala de vidrio utilizada como patrón es un patrón de referencia para instrumental óptico (Figura 9). El paso o distancia entre marcas es la longitud patrón, también el ancho de la marca puede utilizarse como modelo de referencia. En la Tabla 4 se presentan los resultados de la medición, pudiendo observarse gran concordancia entre los valores arrojados por el Rugosímetro y el Microscopio Confocal, aún cuando la calidad metrológica de este último es considerablemente inferior, el resultado es muy adecuado para el uso previsto. El valor obtenido por AFM resulta inconsistente y fuera de tolerancia por un sesgo importante del orden del $3 \%$ respecto del nominal, esto se debe a que el rango máximo de desplazamiento en el plano XY del instrumento es el mismo que la separación de las ranuras impidiendo la determinación adecuada del mismo.

\begin{tabular}{|l|l|}
\hline \multicolumn{2}{|c|}{ Valores del Paso en $\mu \mathrm{m}$} \\
\hline \multicolumn{2}{|c|}{$172-116$} \\
\hline Rugosímetro & $100,0 \pm 0,5$ \\
\hline Confocal & $100 \pm 2$ \\
\hline AFM & $97,7 \pm 0,5$ \\
\hline
\end{tabular}

Tabla 4: Valores del Paso medido con Rugosímetro, Microscopio Confocal y AFM 


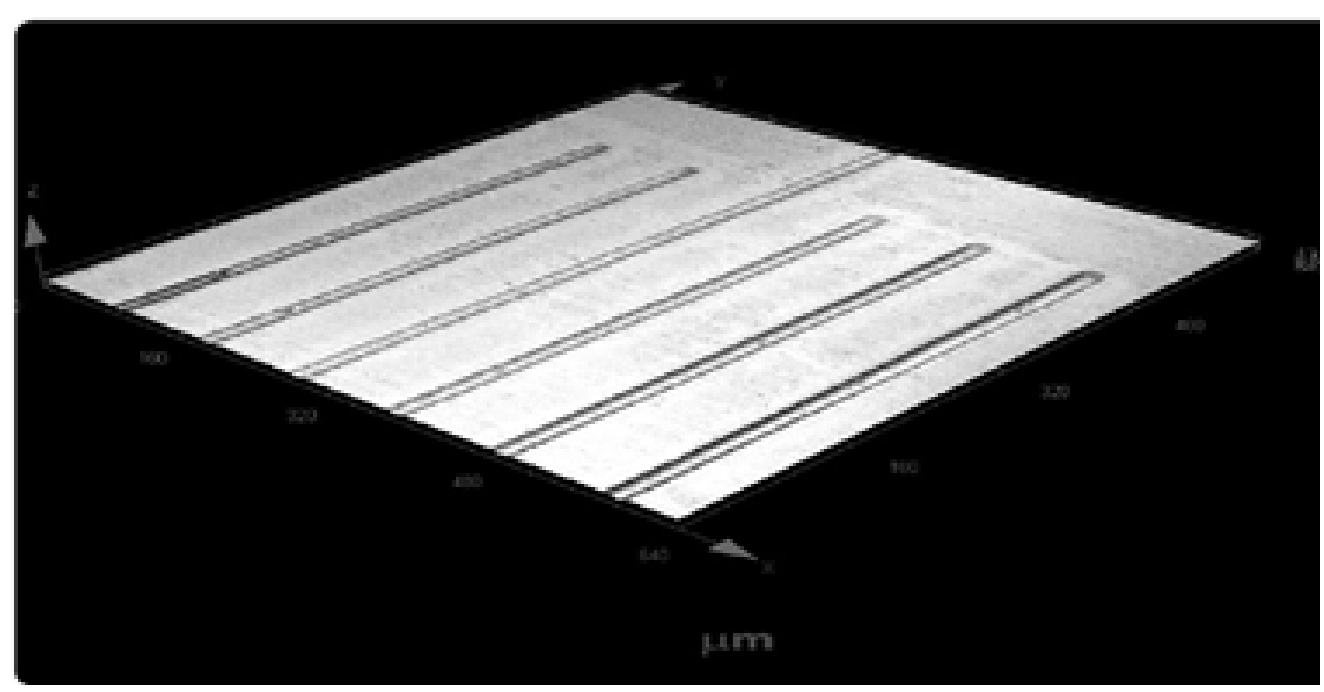

Figura 9: Foto de la escala de vidrio obtenida en Confocal.

\subsection{Patrón de grilla VGRP}

Estas matrices de referencia Bruker Surface Topography tienen una variedad de profundidades y un paso periódico bien determinado; son utilizadas para el control de funcionamiento del AFM, SPM, Confocal y SEM. Los resultados obtenidos se muestran en la Tabla 5. Se observa un muy buen acuerdo tanto en el paso, que son dimensiones en el plano XY, como en la profundidad, una dimensión en el eje Z. En mediciones de profundidad, el AFM es el instrumento más adecuado, con órdenes de incertidumbre muy bajos, es decir con mejor capacidad de medición y mayor exactitud.

La Figura 10 muestra las imágenes y los datos primitivos obtenidos por microscopía.

\begin{tabular}{|l|l|l|}
\hline \multicolumn{3}{|c|}{ Valores de Paso y Profundidad de grilla VGR-18M en $\mu \mathrm{m}$} \\
\hline & Paso & Profundidad \\
\hline Rugosímetro & $10,0 \pm 0,5$ & $0,19 \pm 0,02$ \\
\hline Confocal & $9,9 \pm 0,2$ & $0,2 \pm 0,2$ \\
\hline AFM & $10,12 \pm 0,05$ & $0,199 \pm 0,005$ \\
\hline SEM & $10,1 \pm 0,3$ & - \\
\hline
\end{tabular}

Tabla 5: Valores de Paso y Profundidad de grilla VGRP-18M medido con Rugosímetro, Microscopio Confocal, AFM y SEM 


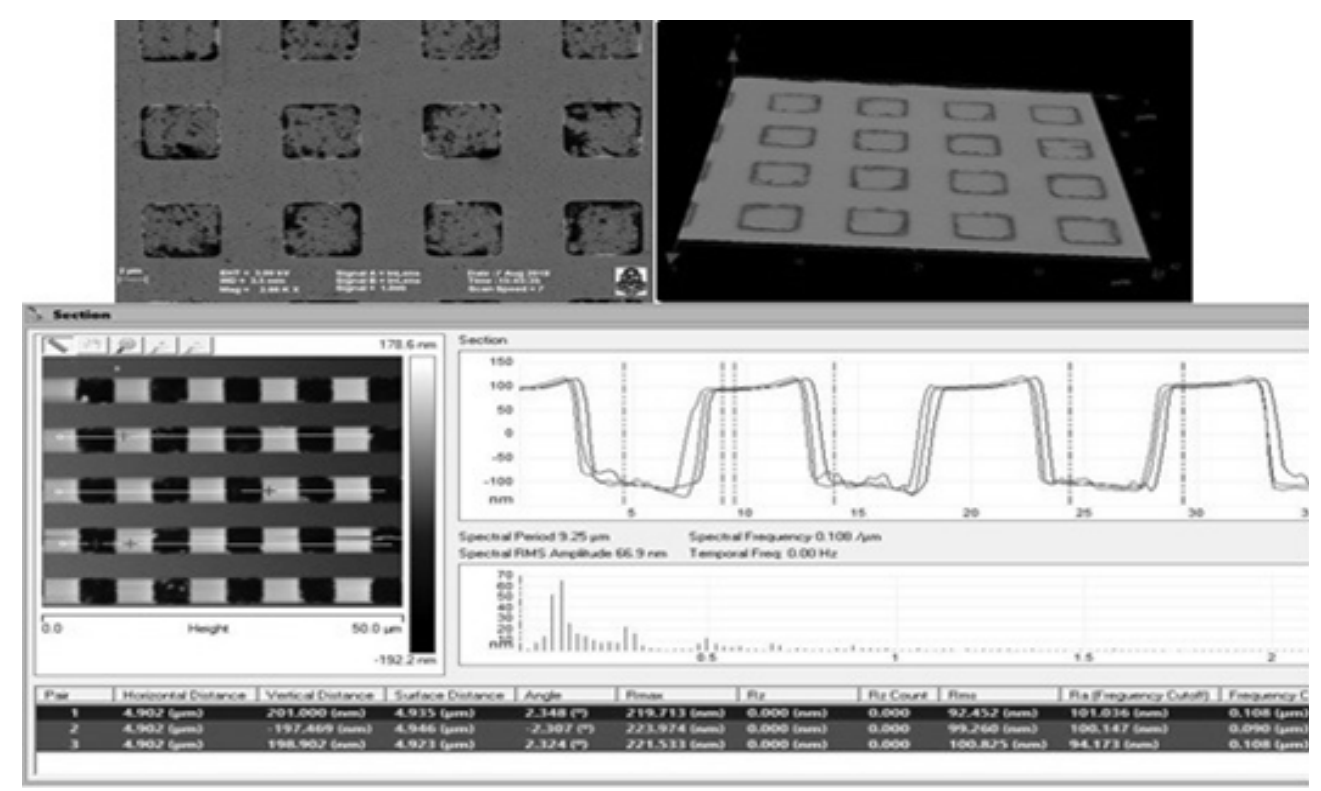

Figura 10: Imagen obtenida con SEM (superior izquierda), con Confocal (Superior derecha) y con AFM (Inferior).

\subsection{Intercomparación de resultados}

Las Tablas 6, 7, 8 y 9 presentan de manera global los resultados del ensayo de intercomparación, a través del índice Error Normalizado "En" de los parámetros característicos de rugosidad relevados con los diferentes instrumentos para cada tipo de patrón y elemento de referencia cuyos detalles se presentaron en el apartado 3. En las Tablas se presenta en cada fila un patrón y su valor nominal y en las columnas subsiguientes los resultados de medición obtenidos sobre el patrón con cada uno de los instrumentos utilizados en el presente trabajo: valor medido promedio e incertidumbre de medición con una probabilidad del 95\% estimada en base a la guía JCGM 100: 2008.

Para el cálculo del "En" se usó como valor de referencia el valor del rugosímetro, que es considerado el Valor Verdadero Convencional, dado que el instrumento está calibrado y tiene trazabilidad a patrones nacionales. En estas condiciones, la práctica puede considerarse como una validación de las mediciones realizadas con los microscopios.

Se comprueba una aceptable coherencia metrológica de los resultados, dando el error normalizado por debajo de uno, lo que implica que el resultado es satisfactorio. Con una excepción, que fue indicada explícitamente en la Tabla 7, consistente con un uso fuera del rango el microscopio AFM, por lo que se descarta.

Las consideraciones respectivas y los hallazgos se fueron explicitando en cada caso y para cada patrón de referencia. 
La robustez del ensayo de intercomparación se respalda en el uso de los criterios, términos y definiciones de la Norma ISO/IEC 17043: Evaluación de Conformidad. Requisitos Generales para los Ensayos de Aptitud, tanto en la sistematización de la preparación y uso de las muestras como en la evaluación estadística de los resultados de cada ensayo, para concluir cuantitativamente sobre la fiabilidad de las mediciones obtenidas con instrumentos de diferente campo de acción y uso sobre los patrones de referencia elegidos.

\begin{tabular}{|c|c|c|c|c|c|c|c|c|c|c|}
\hline \multirow{2}{*}{ Patron } & \multirow{2}{*}{$\begin{array}{l}\text { Valor } \\
\text { Nominal }\end{array}$} & \multicolumn{2}{|c|}{ Federal I.N.T.I. } & \multicolumn{2}{|c|}{$\begin{array}{c}\text { Talysurf Cemetro } \\
\text { UTN }\end{array}$} & \multicolumn{2}{|c|}{$\begin{array}{c}\text { Confocal } \\
\text { Famaf }\end{array}$} & \multirow{2}{*}{\begin{tabular}{|c|}
$\begin{array}{c}\text { Federal L.N.T.I. vs } \\
\text { Talysurf Cemetro UTN }\end{array}$ \\
|En| \\
\end{tabular}} & \multirow{2}{*}{$\begin{array}{c}\begin{array}{c}\text { Federal I.N.T.L. vs } \\
\text { Confocal Famaf }\end{array} \\
|E n| \\
\end{array}$} & \multirow{2}{*}{$\begin{array}{c}\begin{array}{c}\text { Talysurf Cemetro UTN } \\
\text { vs Confocal famaf }\end{array} \\
|E n| \\
\end{array}$} \\
\hline & & \begin{tabular}{|c|} 
Valor \\
Medio $(\mu \mathrm{m})$ \\
\end{tabular} & $\begin{array}{l}\text { U95: } \\
\text { (w/m) }\end{array}$ & \begin{tabular}{|c|} 
Volor \\
Medio (um)
\end{tabular} & $\begin{array}{l}\text { U955 } \\
\text { (amm) }\end{array}$ & \begin{tabular}{|c|} 
Volor \\
Medio $(\mu \mathrm{m})$
\end{tabular} & $\begin{array}{l}\text { U S55 } \\
\text { (mum) }\end{array}$ & & & \\
\hline $\begin{array}{l}\text { Patrón Mitutoyo } \\
\text { (irregular) }\end{array}$ & 0,41 & 0,40 & 0,03 & 0,41 & 0,04 & 0,38 & 0,03 & 0,2 & 0,5 & 0,6 \\
\hline $\begin{array}{l}\text { Patrón Mitutoyo } \\
\text { (irregular) }\end{array}$ & 0,47 & 0,47 & 0,07 & 0,45 & 0,05 & 0,45 & 0,03 & 0,2 & 0,3 & 0,0 \\
\hline $\begin{array}{l}\text { Patrón Mahr } \\
\text { (regular) }\end{array}$ & 1,56 & & & 1,56 & 0,15 & 1,53 & 0,05 & & & 0,2 \\
\hline $\begin{array}{l}\text { Patrón Mitutoyo } \\
\text { (irregular) }\end{array}$ & 2,92 & 2,91 & 0,09 & 2,96 & 0,30 & 2,94 & 0,10 & 0,2 & 0,2 & 0,1 \\
\hline $\begin{array}{l}\text { Patrón Mitutoyo } \\
\text { (irregular) }\end{array}$ & 3,02 & 3,02 & 0,15 & 3,04 & 0,30 & 2,99 & 0,10 & 0,1 & 0,2 & 0,2 \\
\hline
\end{tabular}

Tabla 6: Intercomparación de resultados del Parámetro Ra

\begin{tabular}{|c|c|c|c|c|c|c|c|c|c|c|c|c|}
\hline \multirow{2}{*}{ Patron } & \multirow{2}{*}{$\begin{array}{c}\text { Valor Nominal } \\
(\mu \mathrm{m})\end{array}$} & \multicolumn{2}{|c|}{$\begin{array}{c}\text { Rugosimetro Taylor } \\
\text { Hobson }\end{array}$} & \multicolumn{2}{|c|}{$\begin{array}{c}\text { Confocal Olympus } \\
\text { Lext }\end{array}$} & \multicolumn{2}{|c|}{ AMF Bruker } & \multicolumn{2}{|c|}{ SEM } & \multirow{2}{*}{$\begin{array}{c}\text { Confocal vs Rugosimetro } \\
|E n|\end{array}$} & \multirow{2}{*}{$\begin{array}{c}\text { AMF vs Rugosimetro } \\
\text { |En } \mid\end{array}$} & \multirow{2}{*}{$\begin{array}{c}\text { SEM vs Rugosimetro } \\
\text { En } \mid\end{array}$} \\
\hline & & $\begin{array}{c}\text { Valor Medio } \\
(\mu \mathrm{m})\end{array}$ & $\begin{array}{l}\text { U 95\% } \\
(\mu \mathrm{m})\end{array}$ & $\begin{array}{c}\text { Valor Medio } \\
(\mu \mathrm{m})\end{array}$ & $\begin{array}{l}\text { U 95\% } \\
(\mu \mathrm{m})\end{array}$ & $\begin{array}{c}\text { Valor Medio } \\
(\mu \mathrm{m})\end{array}$ & $\begin{array}{l}\text { U 95\% } \\
(\mu \mathrm{m})\end{array}$ & $\begin{array}{c}\text { Valor Medio } \\
(\mu \mathrm{m})\end{array}$ & $\begin{array}{l}\text { U 95\% } \\
(\mu \mathrm{m})\end{array}$ & & & \\
\hline Grilla VGRP-18M & Paso $10 \mu \mathrm{m}$ & 10,00 & 0,50 & 9,90 & 0,20 & 10,12 & 0,05 & 10,10 & 0,30 & 0,19 & 0,24 & 0,17 \\
\hline Grilla VGRP-18M & \begin{tabular}{|c|} 
Profundidad \\
0,2
\end{tabular} & 0,2 & 0,0 & 0,2 & 0,2 & 0,20 & 0,005 & $\cdot$ & - & 0,05 & 0,44 & - \\
\hline Escala de Vidrio & Paso $100 \mu \mathrm{m}$ & 100,0 & 0,5 & 100,0 & 2,0 & 97,70 & 0,50 & - & - & 0,00 & 3,25 & - \\
\hline
\end{tabular}

Tabla 7: Intercomparación de resultados sobre patrón grilla y escala de vidrio

\begin{tabular}{|c|c|c|c|c|c|c|c|c|c|c|}
\hline \multirow{2}{*}{ Patron } & \multirow{2}{*}{$\begin{array}{c}\text { Valor } \\
\text { Nominal } \\
(\mu \mathrm{m})\end{array}$} & \multicolumn{2}{|c|}{\begin{tabular}{|c|} 
Rugosimetro Taylor \\
Hobson
\end{tabular}} & \multicolumn{2}{|c|}{\begin{tabular}{|c|c|} 
Confocal Olympus \\
Lext
\end{tabular}} & \multicolumn{2}{|c|}{ AMF Bruker } & \multirow{2}{*}{$\begin{array}{c}\begin{array}{c}\text { Confocal Olympus Lext vs } \\
\text { Rugosimetro Taylor Hobson }\end{array} \\
|E n|\end{array}$} & \multirow{2}{*}{\begin{tabular}{|c|}
$\begin{array}{c}\text { AMF Bruker vs Rugosimetro } \\
\text { Taylor Hobson }\end{array}$ \\
|En| \\
\end{tabular}} & \multirow{2}{*}{\begin{tabular}{|c|} 
AMF Bruker vs Confocal \\
Olympus Lext
\end{tabular}} \\
\hline & & $\begin{array}{c}\text { Valor Medio } \\
(\mu \mathrm{m})\end{array}$ & $\begin{array}{l}\text { U 95\% } \\
(\mu \mathrm{m})\end{array}$ & $\begin{array}{c}\text { Valor Medio } \\
(\mu \mathrm{m})\end{array}$ & $\begin{array}{l}\text { U 95\% } \\
(\mu \mu \mathrm{m})\end{array}$ & $\begin{array}{c}\text { Valor Medio } \\
(\mu \mathrm{m})\end{array}$ & $\begin{array}{l}\text { U 95\% } \\
(\mu \mathrm{m})\end{array}$ & & & \\
\hline Ranura de $1 \mu \mathrm{m}$ & 1 & 1,05 & 0,07 & 1,02 & 0,20 & 1,02 & 0,05 & 0,14 & 0,35 & 0,00 \\
\hline Ranura de $4 \mu \mathrm{m}$ & 4 & 3,9 & 0,3 & 3,9 & 0,2 & 3,70 & 0,05 & 0,00 & 0,66 & 0,97 \\
\hline Ranura de $9 \mu \mathrm{m}$ & 9 & 8,9 & 0,6 & 9,2 & 0,3 & - & - & 0,45 & - & - \\
\hline
\end{tabular}

Tabla 8: Intercomparación de resultados sobre patrón de ranura 


\begin{tabular}{|c|c|c|c|c|c|c|c|c|c|c|}
\hline \multirow{2}{*}{ Patron } & \multirow{2}{*}{$\begin{array}{c}\text { Valor } \\
\text { Nominal } \\
(\mu \mathrm{m})\end{array}$} & \multicolumn{2}{|c|}{$\begin{array}{c}\text { Rugosimetro Taylor } \\
\text { Hobson }\end{array}$} & \multicolumn{2}{|c|}{\begin{tabular}{|c|} 
Confocal Olympus \\
Lext
\end{tabular}} & \multicolumn{2}{|c|}{ AMF Bruker } & \multirow{2}{*}{$\begin{array}{c}\begin{array}{c}\text { Confocal Olvmpus lext vs } \\
\text { Rugosimetro Taylor Hobson }\end{array} \\
\mid \text { En } \mid \\
\end{array}$} & \multirow{2}{*}{\begin{tabular}{|c|}
$\begin{array}{c}\text { AMF Bruker vs Rugosimetro } \\
\text { Taylor Hobson }\end{array}$ \\
|En| \\
\end{tabular}} & \multirow{2}{*}{\begin{tabular}{|c|} 
AMF Bruker vs Confocal \\
Olympus Lext
\end{tabular}} \\
\hline & & $\begin{array}{c}\text { Valor Medio } \\
(\mu \mathrm{m})\end{array}$ & $\begin{array}{l}\text { U95\% } \\
(\mu \mathrm{m})\end{array}$ & $\begin{array}{c}\text { Valor Medio } \\
(\mu \mathrm{m})\end{array}$ & $\begin{array}{l}\text { U95\% } \\
(\mu \mathrm{m})\end{array}$ & $\begin{array}{c}\text { Valor Medio } \\
(\mu \mathrm{m})\end{array}$ & 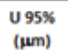 & & & \\
\hline $\begin{array}{c}\text { Patron de } \\
\text { rugosidad PGN3 }\end{array}$ & - & 3,40 & 0,20 & - & - & 3,52 & 0,05 & - & 0,58 & - \\
\hline $\begin{array}{c}\text { Patron de } \\
\text { rugosidad RNDH2 }\end{array}$ & $\cdot$ & 3,4 & 0,2 & - & - & 3,35 & 0,05 & - & 0,24 & - \\
\hline
\end{tabular}

Tabla 9: Intercomparación de resultados del Parámetro Rt

\section{Conclusiones}

En base a los resultados obtenidos en este trabajo se puede concluir que los valores encontrados con los diferentes equipos son comparables, demostrando una consistencia en las mediciones de textura superficial con métodos con y sin contacto y refrendando la potencialidad de los equipos ópticos para futuros trabajos con calidad metrológica, proveyendo la necesaria e importante trazabilidad a patrones nacionales y/o internacionales, requisito imprescindible para la confiabilidad de uso de los instrumentos de laboratorio. Las líneas de acción futuras que se perciben como interesantes para profundizar los estudios empíricos, dar respaldo matemático más riguroso y aplicar los hallazgos son:

-Desarrollo e implementación de procedimientos para calibración de Rugosímetro en CEMETRO. Balance de la incertidumbre.

-Desarrollo e implementación de procedimientos de calibración para el equipo LEXT

-Desarrollo e implementación de procedimientos de medición de rugosidad y calibración para el microscopio de fuerza atómica AFM. Balance de incertidumbre.

-El desarrollo de servicios de Ensayos de Aptitud en base a ISO 17043 para acreditación.

-Acreditación de los servicios de calibración.

-Desarrollo de modelado matemático y validación para la puesta a punto de un método para la calibración de puntas de Rugosímetro por contacto. Este punto surge como novedad de los estudios realizados con el equipo Confocal. 


\section{Referencias}

- C. Jumelle, A. Hamri, G. Eguaud, C. Mauclair, S. Reynaud, V. Dumas, S. Pereira, T. Garcin, P. Gain, G. Thuret. Comparison of four methods of surface roughness assessment of corneal stromal bed after lamellar cutting, BIOMEDICAL OPTICS EXPRESS Vol. 8, No. 11 pp: 4974-4986 (2017)

- David J. Whitehouse. Handbook of surface and nanometrology CRC PressTaylor\&Francis Group 6000 Broken Sound Parkway NW, Suite 300. $2^{\circ}$ ed. 2011

- D J Whitehouse Surface metrology, Meas. Sci. Technol. 8 (1997) 955-972.

- Richard Leach. Measurement Good Practice Guide No. 37. The Measurement of Surface Texture using Stylus Instruments. ISSN 1368-6550 National Physical Laboratory. July 2001 Updated February 2014.

- T.V. Vorburger, Surface finish metrology tutorial, NISTIR 89-4088 (1990).

- N. Jouini, A.Gautier, P. Revel*, P-E. Mazeran and M. Bigerelle, Multi-scale analysis of high precision surfaces by Stylus Profiler, Scanning White-Light Interferometry and Atomic Force Microscopy Int. J. of Surface Science and Engineering Vol.3, No.4 pp.310-327 (2009).

- J. Song, L.Ma, E. Whitenton and T. Vorburger, 2D and 3D Surface Texture Comparisons Using Autocorrelation Functions Key Engineering Materials Vols. 295-296, pp 437-440 (2005)

- Mahesh Chand, Aarti Mehta, Rina Sharma, V N Ojha y K.P. Chaudhary. Roughness measurement using optical profiler with self-reference laser and stylus instrument - A comparative study. Indian Journal of Pure\&Applied Physics Vol. 49, pp. 335-339, 2011.

- Rama Krishna Alla, Kishore Ginjupalli, Nagaraja Upadhya, Mohammed Shammas, Rama Krishna Ravi, Ravichandra Sekhar. Surface Roughness of Implants: A Review. Trends Biomater. Artif. Organs, 25(3), 112-118, (2011)

- F. Ruppa, L. Liang, J. Geis-Gerstorfer, L. Scheideler, F. Hüttig Surface characteristics of dental implants: A review. Dental materials 34 pp. 40-57 (2018).

- Palma D Antonio, Maria Lasalvia, Giuseppe Perna, Vito Capozzi, Scale-independent roughness value of cell membranes studied by means of AFM technique Biochimica et Biophysica Acta 1818 pp. 3141-3148 (2012).

- Jon Petzing, Richard Leach. Measurement Good Practice Guide No.116: The Measurement of Rough Surface Topography using Coherence Scanning Interferometry ISSN 1368-6550 (2010).

- K. Dirscherl and K. R. Koops capítulo 7 en: Nanoscale Calibration Standards and Methods: Dimensional and Related Measurements in the Micro- and Nanometer Range. Edited by Gunter Wilkening, Ludger Koenders. Wiley-VCH Verlag GmbH\& Co. KGaA, Weinheim ISBN: 3-527-40502-X (2005).

- Claudiu L Giusca Richard K Leach Measurement Good Practice Guide No. 128 Calibration of the metrological characteristics of imaging confocal microscopes (ICMs) ISSN 1368-6550 (2012). 
- Richard Leach. The Measurement of Surface Texture using Stylus Instruments Measurement. Good Practice Guide No. 37 (2004) National Physical Laboratory, United Kingdom

- Dirscherl and K.R. Koops Nanoscale calibration, Chapter 7, Traceable Probing with an AFM.

- Measurement Good Practice Guide No.116

- Qi et.al. An unambiguous expression method of the surface textures. Measurement, 43 (10) pp. 1398-1403 de (2010)

- Caracterización de patrones en superficies en micro y nanotecnología con trazabilidad al patrón nacional de longitud. Proyecto Trazasurf. Centro Español de Metrología. 2010.

- Norma ISO 17043 Evaluación de Conformidad - Ensayos de Aptitud.

- Norma ISO 4287 Geometrical Product Specifications (GPS) - Surface texture: Profile method - Terms, definitions and surface texture parameters. 